\title{
EDITORIAL
}

\section{Pentraxin-3 in COPD: innocent bystander or amplifier?}

\author{
A. Mantovani
}

I $\mathrm{n}$ this issue of the European Respiratory Journal, VAN POTTELBERGe et al. [1] report on the association between interstitial levels of the long pentraxin, PTX3, and chronic obstructive pulmonary disease (COPD). COPD remains a formidable challenge in terms of identifying prognostic markers and innovative therapeutic approaches. COPD is characterised by worsening lung function sustained by an unrestrained nonresolving inflammatory response. There is a need for innovative prognostic markers relevant to disease progression and its complications [2]. In this report, VAN PotTelberge et al. [1] found that lower levels of PTX3 in the lung interstitium of COPD patients were associated with airway obstruction as assessed by forced expiratory volume in $1 \mathrm{~s}$ (FEV1). The different levels of PTX3 were not a reflection of different amounts of PTX3 mRNA transcripts, as assessed in whole tissue extracts. Sputum levels were uninformative. Unlike C-reactive protein (CRP), PTX3 levels did not increase in COPD, but a correlation between plasma levels and FEV1 was observed, with a decrease in more advanced disease stages. These findings are unexpected and provocative, raising a number of questions.

PTX3 is a multifunctional protein that acts as a component of the humoral arm of innate immunity [3]. It has antibody-like properties, recognising microbes (e.g. Aspergillus fumigatus, Klebsiella pneumoniae and Pseudomonas aeruginosa), interacting with complement and facilitating phagocytosis [3]. In addition, it serves as a focal point for hyaluronic acid organisation in the extracellular matrix. Unlike its structural relative CRP, it is mainly produced at extrahepatic sites. PTX3 levels increase under inflammatory conditions caused by infection and tissue damage [4-7]. Compared with CRP, PTX3 levels increase more rapidly in pathology, possibly better and directly reflecting tissue inflammation. For instance, PTX3 has been reported to correlate with disease severity in pulmonary infections (e.g. $P$. aeruginosa) and acute respiratory distress syndrome [8-10]. The results reported here in COPD provide an unexpected turn in efforts aimed at exploring the value of PTX3 as a disease marker.

Interstitial PTX3 levels were not correlated with whole tissue mRNA transcripts. This apparent inconsistency may reflect the lack of selection of cells relevant for PTX3 production at the chosen anatomical sites. Alternatively, and more likely, PTX3 levels under these conditions reflect selective degradation or post-transcriptional regulation. In relation to the latter point,

CORRESPONDENCE: A. Mantovani, Istituto Clinico Humanitas IRCCS, University of Milan, Via Manzoni 56, Rozzano, 20089 Milan, Italy. E-mail: alberto.mantovani@humanitasresearch.it we recently found microRNA-mediated regulation of PTX3 (unpublished data).

Inflammation is a key component of the tumour microenvironment [11]. Two pathways link inflammation and cancer. First, activation of oncogenenes that cause cell transformation orchestrates the construction of an inflammatory microenvironment. Secondly, selected inflammatory conditions, involving for instance the gastrointestinal tract and the lung, increase the risk of developing cancer. Components of cancer-related inflammation include the recruitment of leukocytes, tumourassociated macrophages in particular, cytokines and chemokines. Inflammatory cells and their mediators influence fundamental aspects of cancer, including angiogenesis, tumour cell proliferation and survival, invasion and metastasis, and response to therapeutic agents. Thus, inflammation has emerged as a key component of the tumour microenvironment [11]. It is, therefore, not surprising that COPD is associated with increased risk of developing lung cancer. Interestingly, circulating PTX3 has recently emerged as being associated with lung cancer risk [12]. The role, if any, of PTX3 in the COPD-cancer connection remains to be elucidated.

The actual significance of PTX3 in the pathogenesis of COPD and disease progression remains to be elucidated. In mice genetically deficient for PTX3, cigarette smoke-induced emphysema was unaffected [13], arguing against a role in the disease itself. However, PTX3 is an antibody-like molecule essential for resistance against fungi (A. fumigatus), bacteria ( $P$. aeruginosa and K. pneumoniae) and viruses (e.g. influenza) and for apoptotic cell clearance [3]. Moreover, this long pentraxin dampens neutrophil recruitment via interaction with P-selectin [14]. Thus, while not being a prime mover of COPD, available information suggests that decreased lung tissue levels of PTX3 may help disease progression, for instance by compromising an essential component of humoral innate immunity against relevant pathogens.

Circulating levels of PTX3 were within the normal range in this series of COPD patients. PTX3 increases rapidly during infection and is a candidate new marker for disease severity [3, $7,15]$. It remains to be ascertained whether PTX3 may serve as a diagnostic marker of infection during COPD.

The questions raised by the study by VAN POTTELberge et al. [1] will require integrated translational studies in carefully controlled cohorts of patients. In particular, PTX3 polymorphisms have provided genetic evidence in males of the role of this molecule in innate immunity $[8,16,17]$. It will be important to conduct genetic studies to further explore the significance of PTX3 in COPD progression and associated pathology. 


\section{STATEMENT OF INTEREST}

A statement of interest for A. Mantovani can be found at www.erj. ersjournals.com/site/misc/statements.xhtml

\section{REFERENCES}

1 Van Pottelberge GR, Bracke KR, Pauwels NS, et al. COPD is associated with reduced pulmonary interstitial expression of pentraxin-3. Eur Respir J 2012; 39: 830-838.

2 Rabe KF, Hurd S, Anzueto A, et al. Global strategy for the diagnosis, management, and prevention of chronic obstructive pulmonary disease: GOLD executive summary. Am J Respir Crit Care Med 2007; 176: 532-555.

3 Bottazzi B, Doni A, Garlanda C, et al. An integrated view of humoral innate immunity: pentraxins as a paradigm. Annu Rev Immunol 2010; 28: 157-183.

4 Mantovani A, Garlanda C, Doni A, et al. Pentraxins in innate immunity: from C-reactive protein to the long pentraxin PTX3. J Clin Immunol 2008; 28: 1-13.

5 Kaess BM, Vasan RS. Heart failure: pentraxin 3 - a marker of diastolic dysfunction and HF? Nat Rev Cardiol 2011; 8: 246-248.

6 Meier P. Plasma sTWEAK and PTX3: new determinant tools of cardiovascular outcome also in patients with CKD. Clin J Am Soc Nephrol 2011; 6: 697-699.

7 Vänskä M, Koivula I, Hämäläinen S, et al. High pentraxin 3 level predicts septic shock and bacteremia at the onset of febrile neutropenia after intensive chemotherapy of hematologic patients. Haematologica 2011; 96: 1385-1389.
8 Chiarini M, Sabelli C, Melotti P, et al. PTX3 genetic variations affect the risk of Pseudomonas aeruginosa airway colonization in cystic fibrosis patients. Genes Immun 2011; 11: 665-670.

9 Mauri T, Masson S, Pradella A, et al. Elevated plasma and alveolar levels of soluble receptor for advanced glycation endproducts are associated with severity of lung dysfunction in ARDS patients. Tohoku J Exp Med 2010; 222: 105-112.

10 Mauri T, Coppadoro A, Bellani G, et al. Pentraxin 3 in acute respiratory distress syndrome: an early marker of severity. Crit Care Med 2008; 36: 2302-2308.

11 Mantovani A, Allavena P, Sica A, et al. Cancer-related inflammation. Nature 2008; 454: 436-444.

12 Diamandis EP, Goodglick L, Planque C, et al. Pentraxin-3 is a novel biomarker of lung carcinoma. Clin Cancer Res 2011; 17: 2395-2399.

13 Pauwels NS, Bracke KR, Maes T, et al. Cigarette smoke induces PTX3 expression in pulmonary veins of mice in an IL-1 dependent manner. Respir Res 2011; 11: 134.

14 Deban L, Russo RC, Sironi M, et al. Regulation of leukocyte recruitment by the long pentraxin PTX3. Nat Immunol 2010; 11: 328-334.

15 Sica A, Mantovani A. Macrophages in polarized inflammation: in vivo veritas. J Clin Invest 2012; (in press).

16 May L, Kuningas M, van Bodegom D, et al. Genetic variation in pentraxin (PTX) 3 gene associates with PTX3 production and fertility in women. Biol Reprod 2010; 82: 299-304.

17 Olesen R, Wejse C, Velez DR, et al. DC-SIGN (CD209), pentraxin 3 and vitamin $\mathrm{D}$ receptor gene variants associate with pulmonary tuberculosis risk in West Africans. Genes Immun 2007; 8: 456-467. 